\title{
Topology Identification of Directed Dynamical Networks via Power Spectral Analysis
}

\author{
Shahin Shahrampour and Victor M. Preciado, Member, IEEE,
}

\begin{abstract}
We address the problem of identifying the topology of an unknown weighted, directed network of LTI systems stimulated by wide-sense stationary noises of unknown power spectral densities. We propose several reconstruction algorithms based on the cross-power spectral densities of the network's response to the input noises. Our first algorithm reconstructs the Boolean structure (i.e., existence and directions of links) of a directed network from a series of dynamical responses. Moreover, we propose a second algorithm to recover the exact structure of the network (including edge weights), as well as the power spectral density of the input noises, when an eigenvalue-eigenvector pair of the connectivity matrix is known (for example, Laplacian connectivity matrices). Finally, for the particular cases of nonreciprocal networks (i.e., networks with no directed edges pointing in opposite directions) and undirected networks, we propose specialized algorithms that result in a lower computational cost.
\end{abstract}

\section{INTRODUCTION}

The reconstruction of networks of dynamical systems is an important task in many realms of science and engineering, including biology [1] $-[4]$, physics [5]-[7] and finance [8]. In the literature, we find a wide collection of approaches aiming to solve the network reconstruction problem. In the physics literature, we find in [6] a method to identify a network of dynamical systems which assumes that the input of each node can be individually manipulated. In [10], an approach based on Granger's causality [11] and the theory of reproducing kernel Hilbert spaces is proposed. In the statistics community, the reconstruction problem is usually approached using graphical models by associating a random variable to each node and assuming that the (vectorvalued) observations are independent and identically distributed. In this setting, Bach and Jordan Pennsylvania, Philadelphia, PA 19104 USA. (e-mail: shahin@seas.upenn.edu; preciado@ seas.upenn.edu). 
[12] used the Bayesian information criterion (BIC) to estimate sparse graphs from stationary time series. The optimization community has recently proposed a collection of papers approaching the reconstruction problem [4], [7], [13]. In these papers, several optimization problem are proposed to find the sparsest network given a priori structural information. Although the assumption of sparsity is well justified in some applications (e.g. biological networks), this assumptions might lead to unsuccessful topology inference in other cases, as illustrated in [14] and [15]. For tree networks, several techniques for reconstruction were proposed in [8], [16] and [17]. More recently, Materassi and Salapaka proposed in [18] a methodology for reconstruction of directed networks using locality properties of the Wiener filters. In [19], [20], Nabi-Abdolyousefi and Mesbahi proposed techniques to extract structural information of an undirected network running consensus dynamics.

In this paper, we propose several algorithms to reconstruct the structure of a directed network interconnecting a collection of linear dynamical systems. We first propose an algorithm to find the Boolean structure of the unknown topology. This algorithm is based on the analysis of power spectral properties of the network response when the inputs are wide-sense stationary (WSS) processes of unknown power spectral density (PSD). Apart from recovering the Boolean structure of the network, we propose another algorithm to recover the exact structure of the network (including edge weights) when an eigenvalue-eigenvector pair of the connectivity matrix is known. This algorithm can be applied, for example, in the case of the connectivity matrix being a Laplacian matrix or the adjacency of a regular graph. Apart from general directed networks, we also propose reconstruction methodologies for directed nonreciprocal networks (networks with no directed edges pointing in opposite directions) and undirected networks. In the latter cases, we propose specialized algorithms able to recover the network structure with less computational cost.

The rest of the paper is organized as follows. In section II, we introduce some preliminary definitions needed in our exposition and describe the network reconstruction problem under consideration. Section III provides several theoretical results that are the foundation for our reconstruction techniques. In Section IV, we introduce several algorithms to reconstruct the Boolean structure of a directed network (Section IV.A), the exact structure of a directed network given an eigenvalue-eigenvector pair (Section IV.B), and the structure of undirected and nonreciprocal networks (Sections IV.C and IV.D, respectively). We finish with some conclusions in Section V. 
Nomenclature

$I_{d} \quad d \times d$ identity matrix.

$\mathbf{1}_{d} \quad d$-dimensional vector of all ones.

$\mathbb{E}(\cdot) \quad$ Expectation operator.

$R_{x y}(\tau) \quad$ Cross-correlation function, $\mathbb{E}(x(t) y(t-\tau))$.

$R_{x}(\tau) \quad$ Auto-correlation function, $\mathbb{E}(x(t) x(t-\tau))$.

$\mathcal{F}\{\cdot\} \quad$ Fourier transform.

$S_{y_{i} y_{j}}(\omega) \quad$ Cross-power spectral density (CPSD), $\mathcal{F}\left\{R_{y_{i} y_{j}}(\tau)\right\}$.

$S_{y_{i}}(\omega) \quad$ Power spectral density (PSD), $\mathcal{F}\left\{R_{y_{i} y_{i}}(\tau)\right\}$.

\section{Preliminaries \& Problem Description}

In this section, we state the problem under consideration. First, we introduce some notions of graph theory which are needed in our derivations (see [21], for an extensive exposition).

\section{A. Graph Theory}

Let $\mathcal{G} \triangleq(\mathcal{V}, \mathcal{E})$ be an unweighted, undirected graph, where $\mathcal{V} \triangleq\left\{v_{1}, \ldots, v_{N}\right\}$ denotes a set of $N$ nodes and $\mathcal{E} \subseteq \mathcal{V} \times \mathcal{V}$ denotes a set of $m$ undirected edges. If $\left\{v_{i}, v_{j}\right\} \in \mathcal{E}$, we call nodes $v_{i}$ and $v_{j}$ adjacent (or first-neighbors), which we denote by $v_{i} \sim v_{j}$. A weighted, undirected graph is defined as the triad $\mathcal{W} \triangleq(\mathcal{V}, \mathcal{E}, \mathcal{F})$, where $\mathcal{V}$ and $\mathcal{E}$ are the sets of nodes and edges in $\mathcal{W}$, and the function $\mathcal{F}: \mathcal{E} \rightarrow \mathbb{R}$ associates real weights to the edges. Similarly, a weighted, directed graph is defined as the triad $\mathcal{D} \triangleq\left(\mathcal{V}, \mathcal{E}_{d}, \mathcal{F}_{d}\right)$, where $\mathcal{V}$ is the set of nodes and $\mathcal{E}_{d}$ is the set of directed edges in $\mathcal{D}$, where a directed edge from node $v_{i}$ to node $v_{j}$ is defined as an ordered pair $\left(v_{i}, v_{j}\right)$. Furthermore, $\mathcal{F}_{d}$ is a weight function $\mathcal{F}_{d}: \mathcal{E}_{d} \rightarrow \mathbb{R}$.

In an unweighted, undirected graph $\mathcal{G}$, the degree of a vertex $v_{i}$, denoted by $\operatorname{deg}\left(v_{i}\right)$, is the number of nodes adjacent to it, i.e., $\operatorname{deg}\left(v_{i}\right)=\left|\left\{v_{j} \in \mathcal{V}:\left\{v_{i}, v_{j}\right\} \in \mathcal{E}\right\}\right|$. This definition can be generalized to both weighted and directed graphs. For weighted graphs, the weighted degree of node $v_{i}$ is equal to $\operatorname{deg}\left(v_{i}\right)=\sum_{j:\left\{v_{i}, v_{j}\right\} \in \mathcal{E}} \mathcal{F}\left(\left\{v_{i}, v_{j}\right\}\right)$, i.e., the sum of the weights associated to edges connected to $v_{i}$. For weighted, directed networks, we define the weighted in-degree of node $v_{i}$ as $\operatorname{deg}_{i n}\left(v_{i}\right)=\sum_{j:\left(v_{j}, v_{i}\right) \in \mathcal{E}_{d}} \mathcal{F}_{d}\left(\left(v_{j}, v_{i}\right)\right)$.

The adjacency matrix of an unweighted, undirected graph $\mathcal{G}$, denoted by $A_{\mathcal{G}}=\left[a_{i j}\right]$, is a $N \times N$ Boolean symmetric matrix defined entry-wise as $a_{i j}=1$ if nodes $v_{i}$ and $v_{j}$ are adjacent, 
and $a_{i j}=0$ otherwise. We define the Laplacian matrix $L_{\mathcal{G}}$ of a graph $\mathcal{G}$ as $L_{\mathcal{G}}=D_{\mathcal{G}}-A_{\mathcal{G}}$ where $D_{\mathcal{G}}$ is the diagonal matrix of degrees, $D_{\mathcal{G}}=\operatorname{diag}\left(\left(\operatorname{deg}\left(v_{i}\right)\right)_{i=1}^{N}\right)$. For simple graphs, $L_{\mathcal{G}}$ is a symmetric positive semidefinite matrix, which we denote by $L_{\mathcal{G}} \succeq 0$ [22]. Thus, $L_{\mathcal{G}}$ has a full set of $N$ real and orthogonal eigenvectors with real nonnegative eigenvalues $0=\lambda_{1} \leq \lambda_{2} \leq \ldots \leq \lambda_{N}$.

Similarly, the weighted adjacency matrix of a weighted graph $\mathcal{W}$ is defined as $A_{\mathcal{W}}=\left[w_{i j}\right]$, where $w_{i j}=\mathcal{F}\left(\left\{v_{i}, v_{j}\right\}\right)$ for $\left\{v_{i}, v_{j}\right\} \in \mathcal{E}$, and $w_{i j}=0$ if $\left\{v_{i}, v_{j}\right\} \notin \mathcal{E}$. We define the degree matrix of a weighted graph $\mathcal{W}$ as the diagonal matrix $D_{\mathcal{W}}=\operatorname{diag}\left(\left(\operatorname{deg}\left(v_{i}\right)\right)_{i=1}^{N}\right)$. The Laplacian matrix of a weighted, undirected graph $\mathcal{W}$, is defined as $L_{\mathcal{W}}=D_{\mathcal{W}}-A_{\mathcal{W}}$. Furthermore, the adjacency matrix of a weighted, directed graph $\mathcal{D}$ is defined as $A_{\mathcal{D}}=\left[d_{i j}\right]$, where $d_{i j}=\mathcal{F}_{d}\left(\left(v_{j}, v_{i}\right)\right)$ for $\left(v_{j}, v_{i}\right) \in \mathcal{E}_{d}$, and $d_{i j}=0$ if $\left(v_{j}, v_{i}\right) \notin \mathcal{E}_{d}$. We define the in-degree matrix of a directed graph $\mathcal{D}$ as the diagonal matrix $D_{\mathcal{D}}=\operatorname{diag}\left(\left(\operatorname{deg}_{i n}\left(v_{i}\right)\right)_{i=1}^{N}\right)$. The Laplacian matrix of $\mathcal{D}$ is then defined as $L_{\mathcal{D}}=D_{\mathcal{D}}-A_{\mathcal{D}}$. The Laplacian matrix, for all the unweighted, weighted, and directed cases, satisfies $L_{\mathcal{G}} \mathbf{1}=L_{\mathcal{W}} \mathbf{1}=L_{\mathcal{D}} \mathbf{1}=\mathbf{0}$, i.e., the vector $\mathbf{1} / \sqrt{N}$ is an eigenvector of the Laplacian matrix with eigenvalue 0.

\section{B. Dynamical Network Model \& Problem Statement}

Consider a dynamical network consisting of $N$ linearly coupled identical nodes, with each node being an $n$-dimensional LTI SISO dynamical system. The dynamical network under study can be characterized by

$$
\begin{aligned}
& \dot{x}_{i}(t)=A x_{i}(t)+b\left(\sum_{j=1}^{N} g_{i j} y_{j}(t)+w_{i}(t)\right), \\
& y_{i}(t)=c^{T} x_{i}(t),
\end{aligned}
$$

where $x_{i}(t) \in \mathbb{R}^{n}$ denotes the state vector describing the dynamics of node $v_{i} \in \mathcal{V} . A \in \mathbb{R}^{n \times n}$ and $b, c \in \mathbb{R}^{n}$ are the given state, input and output matrices corresponding to the state-space representation of each node in isolation. $w_{i}(t)$ and $y_{i}(t) \in \mathbb{R}$ are stochastic processes representing the input noise and the system output, respectively. $g_{i j} \geq 0$ is the coupling strength of a directed edge from $v_{i}$ to $v_{j}$, which we shall assume to be unknown.

Defining the network state vector $\mathbf{x}(t) \triangleq\left(x_{1}^{T}(t), \ldots, x_{N}^{T}(t)\right)^{T} \in \mathbb{R}^{N n}$, the noise vector $\mathbf{w}(t) \triangleq$ $\left(w_{1}(t), \ldots, w_{N}(t)\right)^{T} \in \mathbb{R}^{N}$, and the network output vector $\mathbf{y}(t) \triangleq\left(y_{1}(t), \ldots, y_{N}(t)\right)^{T} \in \mathbb{R}^{N}$, 
respectively, we can rewrite the network dynamics in (1), as

$$
\begin{aligned}
& \dot{\mathbf{x}}(t)=\left(I_{N} \otimes A+\mathbf{G} \otimes b c^{T}\right) \mathbf{x}(t)+\left(I_{N} \otimes b\right) \mathbf{w}(t), \\
& \mathbf{y}(t)=\left(I_{N} \otimes c^{T}\right) \mathbf{x}(t),
\end{aligned}
$$

where $\mathbf{G}=\left[g_{i j}\right]$ is the connectivity matrix of a (possibly weighted and/or directed) network $\mathcal{D}$. For the networked dynamical system to be stable, we assume the network state matrix $I_{N} \otimes A+$ $\mathbf{G} \otimes b c^{T}$ to be Hurwitz.

Hereafter, we will analyze the following scenario. Consider a collection of $N$ dynamical nodes with a known LTI SISO dynamics defined by the state-space matrices $\left(A, b, c^{T}, 0\right)$. The link structure of the network dynamic model, described by the connectivity matrix $\mathrm{G}$, is completely unknown. We assume the input noises, $w_{i}(t)$, are i.i.d. wide-sense stationary processes of unknown but identical power spectral densities, i.e., $S_{w_{i}}(\omega)=S_{w}(\omega)$ for all $i=1, \ldots, N$. We are interested in identifying all the links in the network by exploiting only the information provided by the realizations of the output stochastic processes $y_{1}(t), \ldots, y_{N}(t)$. Formally, we can formulate this problem as follows:

Problem 1: Consider the dynamical network model in (2), whose connectivity matrix G is unknown. Assume that the only available information is a spectral characterization of the output signals $y_{1}(t), \ldots, y_{N}(t)$ in terms of power and cross-power spectral densities, $S_{y_{i}}(\omega)$ and $S_{y_{i} y_{j}}(\omega)$, which can be empirically estimated from the output signald 1 . Then, find the Boolean structure of the directed network, i.e., the location and directions of all the edges.

It is worth remarking that we assume the input noise to be an exogenous signal of unknown power spectral density, $S_{w}(\omega)$. We will provide in Section IV-A a methodology to recover the Boolean structure of the network, even though the input noise is not known. We will show in Section IV-B that for certain connectivity matrices, such as Laplacian matrices, we can recover the weights of the directed edges in the network, as well as the power spectral density of the input noise. Moreover, in Sections IV-C and IV-D we provide two computationally efficient algorithms to recover the structure of undirected and nonreciprocal networks, respectively.

\footnotetext{
${ }^{1}$ One can use, for example, Bartletts averaging method [23] to produce periodogram estimates of power and cross-power spectral densities, $S_{y_{i}}(\omega)$ and $S_{y_{i} y_{j}}(\omega)$.
} 


\section{Theoretical Results}

We start by stating some assumptions we need in our subsequent developments. The following definition will be useful for determining sufficient conditions for detection of links in a network.

Definition 2: [Excitation Frequency Interval, [18]] The excitation frequency interval of a vector $\mathbf{w}(t)$ of wide-sense stationary processes is defined as an interval $(-\Omega, \Omega)$, with $\Omega>0$, such that the power spectral densities of the input components $w_{i}(t)$ satisfy $S_{w_{i}}(\omega)>0$ for all $\omega \in(-\Omega, \Omega)$, and all $i \in\{1,2, \ldots, N\}$.

Throughout the paper we impose the following conditions on the input vector:

A1. The collection of signals $\left\{w_{i}(t), i=1, \ldots, N\right\}$ are uncorrelated zero-mean WSS processes with identical autocorrelation function, i.e., for any $t, \tau \in \mathbb{R}, R_{w_{i}}(\tau)=\mathbb{E}\left(w_{i}(t) w_{i}(t+\right.$ $\tau)) \triangleq R_{w}(\tau)$.

A2. The input noise $\mathbf{w}(t)$ presents a nonempty excitation frequency interval $(-\Omega, \Omega)$. In our derivations, we will invoke the following variation of the matrix inversion lemma [24]:

Lemma 3 (Sherman-Morrison-Woodbury): Assume that the matrices $D$ and $I+W D^{-1} U E$ are nonsingular. Then, the following identity holds

$$
(D+U E W)^{-1}=D^{-1}-D^{-1} U E\left(I+W D^{-1} U E\right)^{-1} W D^{-1}
$$

where $E, W, D$, and $U$ are matrices of compatible dimensions and $I$ is the identity matrix.

Based on Woodbury's formula, we derive an expression that provides an explicit relationship between the (cross-)power spectral densities of two stochastic outputs, $y_{i}(t)$ and $y_{j}(t)$, when we inject a noise $w_{k}(t)$ into node $k$ with power spectral density $S_{w}(\omega)$.

Lemma 4: Consider the continuous-time networked dynamical system (2). Then, under assumptions (A1)-(A2), the following identity holds

$$
\mathbf{S}(\omega)=S_{w}(\omega)\left(\frac{I_{N}}{|h(\mathbf{j} \omega)|^{2}}+\mathbf{G}^{T} \mathbf{G}-\frac{\mathbf{G}}{h^{*}(\mathbf{j} \omega)}-\frac{\mathbf{G}^{T}}{h(\mathbf{j} \omega)}\right)^{-1}
$$

where $\mathbf{S}(\omega) \triangleq\left[S_{y_{i} y_{j}}(\omega)\right]$ is the matrix of output CPSD's, and $h(\mathbf{j} \omega) \triangleq c^{T}\left(\mathbf{j} \omega I_{n}-A\right)^{-1} b$ is the nodal transfer function. 
Proof: The $N \times N$ transfer matrix, $H(\mathbf{j} w) \triangleq\left[H_{j i}(\mathbf{j} \omega)\right]$, of the state-space model in (2) is given by

$$
\begin{aligned}
H(\mathbf{j} \omega) & =\left(I_{N} \otimes c^{T}\right)\left(\mathbf{j} \omega I_{N n}-I_{N} \otimes A-\mathbf{G} \otimes b c^{T}\right)^{-1}\left(I_{N} \otimes b\right) \\
& =\left(I_{N} \otimes c^{T}\right)\left(I_{N} \otimes\left(\mathbf{j} \omega I_{n}-A\right)-\mathbf{G} \otimes b c^{T}\right)^{-1}\left(I_{N} \otimes b\right) .
\end{aligned}
$$

Assume we inject a noise signal into the $k$-th node, i.e., $\mathbf{w}(t)=w_{k}(t) \mathbf{e}_{k}$. Hence, the power spectral density measured on the output of node $i$ is equal to $S_{y_{i}}(\omega)=H_{k i}(\omega) H_{k i}^{*}(\omega) S_{w_{k}}(\omega)$. On the other hand, the transfer functions from input $w_{k}(t)$ to the outputs $y_{i}(t)$ and $y_{j}(t)$ are, respectively, $Y_{i}(\mathbf{j} \omega) / W_{k}(\mathbf{j} \omega)=H_{k i}(\mathbf{j} \omega)$ and $Y_{j}(\mathbf{j} \omega) / W_{k}(\mathbf{j} \omega)=H_{k j}(\mathbf{j} \omega)$, where $Y_{i}(\mathbf{j} \omega)$ and $W_{k}(\mathbf{j} \omega)$ are the Fourier transforms of $y_{i}(t)$ and $w_{k}(t)$, respectively. Hence, $Y_{j}(\mathbf{j} \omega) / Y_{i}(\mathbf{j} \omega)=$ $H_{k i}^{-1}(\mathbf{j} \omega) H_{k j}(\mathbf{j} \omega)$ which implies $S_{y_{i} y_{j}}(\omega)=\left(H_{k j}(\mathbf{j} \omega) H_{k i}^{-1}(\mathbf{j} \omega)\right)^{*} S_{y_{i}}(\omega)$. Since $S_{w_{k}}(\omega)=S_{w}(\omega)$ for all $k$, we have that $S_{y_{i} y_{j}}(\omega)=H_{k i}(\mathbf{j} \omega) H_{k j}^{*}(\mathbf{j} \omega) S_{w}(\omega)$.

Assume that we inject noise signals satisfying assumptions (A1)-(A2) into all the nodes in the network, i.e., $\mathbf{w}(t)=\sum_{k=1}^{N} w_{k}(t) \mathbf{e}_{k}$. Hence, we can apply superposition to obtain

$$
\begin{aligned}
\frac{S_{y_{i} y_{j}}(\omega)}{S_{w}(\omega)} & =\sum_{k=1}^{N} H_{k j}^{*}(\mathbf{j} \omega) H_{k i}(\mathbf{j} \omega) \\
& =\sum_{k=1}^{N} \mathbf{e}_{k}^{T} H^{*}(\mathbf{j} \omega) \mathbf{e}_{j} \mathbf{e}_{i}^{T} H(\mathbf{j} \omega) \mathbf{e}_{k} \\
& =\sum_{k=1}^{N} \operatorname{Tr}\left(H^{*}(\mathbf{j} \omega) \mathbf{e}_{j} \mathbf{e}_{i}^{T} H(\mathbf{j} \omega) \mathbf{e}_{k} \mathbf{e}_{k}^{T}\right) \\
& =\operatorname{Tr}\left(H^{*}(\mathbf{j} \omega) \mathbf{e}_{j} \mathbf{e}_{i}^{T} H(\mathbf{j} \omega) \sum_{k=1}^{N} \mathbf{e}_{k} \mathbf{e}_{k}^{T}\right) \\
& =\mathbf{e}_{i}^{T} H(\mathbf{j} \omega) H^{*}(\mathbf{j} \omega) \mathbf{e}_{j},
\end{aligned}
$$

for any $\omega \in(-\Omega, \Omega)$, where we used the identity $\sum_{k=1}^{N} \mathbf{e}_{k} \mathbf{e}_{k}^{T}=I_{N}$ in our derivation.

Let us define the matrices $W \triangleq I_{N} \otimes c^{T}, U \triangleq I_{N} \otimes b, E \triangleq-\mathbf{G}$, and $D \triangleq I_{N} \otimes\left(\mathbf{j} \omega I_{n}-A\right)$. Then, we can rewrite the transfer matrix $H(\mathbf{j} \omega)$ in (4) as

$$
H(\mathbf{j} \omega)=W(D+U E W)^{-1} U \text {. }
$$


Also, we have that $h(\mathbf{j} \omega) I_{N}=W D^{-1} U$. Then, applying Lemma 3 to (6), we can rewrite the transfer matrix, as follows

$$
\begin{aligned}
H(\mathbf{j} \omega) & =h(\mathbf{j} \omega)\left(I_{N}+\mathbf{G}\left(I_{N}-h(\mathbf{j} \omega) \mathbf{G}\right)^{-1} h(\mathbf{j} \omega) I_{N}\right) \\
& =h(\mathbf{j} \omega)\left(I_{N}+\mathbf{G}\left(\frac{I_{N}}{h(\mathbf{j} \omega)}-\mathbf{G}\right)^{-1}\right) \\
& =h(\mathbf{j} \omega)\left(I_{N}+\left(\mathbf{G}-\frac{I_{N}}{h(\mathbf{j} \omega)}+\frac{I_{N}}{h(\mathbf{j} \omega)}\right)\left(\frac{I_{N}}{h(\mathbf{j} \omega)}-\mathbf{G}\right)^{-1}\right) \\
& =h(\mathbf{j} \omega)\left(I_{N}-I_{N}+\frac{1}{h(\mathbf{j} \omega)}\left(\frac{I_{N}}{h(\mathbf{j} \omega)}-\mathbf{G}\right)^{-1}\right) \\
& =\left(\frac{I_{N}}{h(\mathbf{j} \omega)}-\mathbf{G}\right)^{-1}
\end{aligned}
$$

Substituting the above into (5), we reach the statement of our lemma after a simple expansion of the resulting expression.

In the following section, we will use this lemma to reconstruct an unknown network structure $\mathrm{G}$ from the empirical CPSD's of the outputs. We will also show that, assuming that we know one eigenvalue-eigenvector pair of $\mathrm{G}$, we can recover the weighted and directed graph $\mathcal{D}$ (not only its Boolean structure), as well as the PSD of the noise, $S_{w}(\omega)$. Relevant examples of this situation are: (i) networks of diffusively coupled systems with a Laplacian connectivity matrix [25], i.e., $\mathbf{G}=-L_{\mathcal{D}}$, since Laplacian matrices always satisfy $L_{\mathcal{D}} \mathbf{1}_{N}=0$; or (ii) $k$-regular networks [21], i.e., $\mathbf{G}=A_{k}$, since the adjacency matrix $A_{k}$ satisfy $A_{k} \mathbf{1}_{N}=k$.

As stated in Problem 1, the PSD of the input noise $\mathbf{w}(t)$ is not available to us to perform the network reconstruction. The following lemma will allow us reconstruct this PSD when an eigenvalue-eigenvector pair of $\mathrm{G}$ is known a priori.

Lemma 5: Consider the continuous-time networked dynamical system (2). Then, under assumptions (A1)-(A2), the input PSD can be computed as

$$
S_{w}(\omega)=\frac{\lambda^{2}|h(\mathbf{j} \omega)|^{2}-2 \lambda \operatorname{Re}\{h(\mathbf{j} \omega)\}+1}{\left(\boldsymbol{u}^{T} \mathbf{S}^{-1}(\omega) \boldsymbol{u}\right)|h(\mathbf{j} \omega)|^{2}},
$$

where $(\lambda, \boldsymbol{u})$ is an eigenvalue-eigenvector pair of $\mathbf{G}, h(\mathbf{j} \omega)$ is the nodal transfer function, and $\mathbf{S}(\omega) \triangleq\left[S_{y_{i} y_{j}}(\omega)\right]$ is the matrix of CPSD's. 
Proof: From (3), we have

$$
\mathbf{S}^{-1}(\omega) S_{w}(\omega)=\frac{I_{N}}{|h(\mathbf{j} \omega)|^{2}}+G^{T} G-\frac{G}{h^{*}(\mathbf{j} \omega)}-\frac{G^{T}}{h(\mathbf{j} \omega)} .
$$

Pre- and post-multiplying by $\boldsymbol{u}^{T}$ and $\boldsymbol{u}$, respectively, we obtain

$$
\left(\boldsymbol{u}^{T} \mathbf{S}^{-1}(\omega) \boldsymbol{u}\right) S_{w}(\omega)=\frac{1}{|h(\mathbf{j} \omega)|^{2}}+\lambda^{2}-\frac{\lambda}{h(\mathbf{j} \omega)}-\frac{\lambda}{h^{*}(\mathbf{j} \omega)}
$$

Dividing by $\boldsymbol{u}^{T} \mathbf{S}^{-1}(\omega) \boldsymbol{u}$, we reach (7).

Lemma 5 shows that, given the eigenvalue-eigenvector pair $(\lambda, \boldsymbol{u})$, the PSD of the input noise can be reconstructed from the nodal transfer function and the matrix of CPSD's, $\mathbf{S}(\omega)$, which can be numerically approximated from the empirical cross-correlations between output signals.

\section{Reconstruction Methodologies}

Based on the above results, we introduce several methodologies to reconstruct the structure of an unknown network following the dynamics in (2) when the PSD of the input noise is unknown. First, in Subsection IV-A, we present a technique to reconstruct the Boolean structure of an unknown (possibly weighted) directed network. Moreover, if an eigenvalue-eigenvector pair of $\mathbf{G}$ is known (for example, $\mathrm{G}$ is a Laplacian matrix), we show how to recover the weights of the directed edges, as well as the PSD of the input noise in Subsection IV-B. Finally, in Subsections IV-C and IV-D we provide reconstruction techniques to recover two special cases, namely, undirected networks and nonreciprocal directed networks, respectively.

Consider Problem 1, when G is an unknown connectivity matrix representing a weighted, directed network $\mathcal{D}$. We propose a reconstruction technique to recover the Boolean structure of $\mathcal{D}$ when the PSD of the input noise is unknown. Note that, in general, the result in Lemma 4 is not enough to extract the underlying structure of the network, even if the input noise PSD were known. In what follows, we propose a methodology to reconstruct a directed network of dynamical nodes by grounding the dynamics in a series of nodes, similar to the approach proposed in [20] to reconstruct undirected networks following a consensus dynamics. 
Definition 6 (Grounded Dynamics): The dynamics of (2) grounded at node $v_{j}$ takes the form

$$
\begin{aligned}
\dot{\widetilde{\mathbf{x}}}(t) & =\left(I_{N-1} \otimes A+\widetilde{\mathbf{G}}_{j} \otimes b c^{T}\right) \widetilde{\mathbf{x}}(t)+\left(I_{N-1} \otimes b\right) \widetilde{\mathbf{w}}(t), \\
\widetilde{\mathbf{y}}(t) & =\left(I_{N-1} \otimes c^{T}\right) \widetilde{\mathbf{x}}(t),
\end{aligned}
$$

where $\widetilde{\mathbf{w}}(t)$ is obtained by eliminating the $j$-th entry from the noise input $\mathbf{w}(t)$, and $\widetilde{\mathbf{G}}_{j} \in$ $\mathbb{R}^{(N-1) \times(N-1)}$ is obtained by eliminating the $j$-th row and column from $\mathbf{G}$.

The dynamics in (8) describes the evolution of (2) when we ground the state of node $v_{j}$ to be $x_{j}(t) \equiv 0$. Applying Lemma 4 to the grounded dynamics (8), one obtains the following expression for the CPSD's:

$$
\widetilde{\mathbf{S}}_{j}(\omega)=S_{w}(\omega)\left(\frac{I_{N}}{|h(\mathbf{j} \omega)|^{2}}+\widetilde{\mathbf{G}}_{j}^{T} \widetilde{\mathbf{G}}_{j}-\frac{\widetilde{\mathbf{G}}_{j}}{h^{*}(\mathbf{j} \omega)}-\frac{\widetilde{\mathbf{G}}_{j}^{T}}{h(\mathbf{j} \omega)}\right)^{-1} .
$$

We will use the next Theorem to propose several reconstruction techniques in Subsections IV-A and IV-B.

Theorem 7: Consider the networked dynamical system (2) with connectivity matrix $\mathbf{G}=\left[g_{i j}\right]$. Let us denote by $S_{w}(\omega)$ the PSD of the input noise, by $\mathbf{S}(\omega)=\left[S_{y_{i} y_{j}}(\omega)\right]$ the $N \times N$ matrix of CPSD's for the (ungrounded) dynamics (2), and by $\widetilde{\mathbf{S}}_{j}(\omega)=\left[\widetilde{S}_{y_{i} y_{k}}(\omega)\right]_{i, k \neq j}$ the $N-1 \times N-1$ matrix of CPSD's for the dynamics in (8) grounded at node $v_{j}$. Then, under assumptions (A1)(A2), we have that, for any $\omega_{0} \in(-\Omega, \Omega)$,

$$
g_{j i}= \begin{cases}{\left[S_{w}\left(\omega_{0}\right)\left(\left[\mathbf{S}^{-1}\left(\omega_{0}\right)\right]_{i i}-\left[\widetilde{\mathbf{S}}_{j}^{-1}\left(\omega_{0}\right)\right]_{i i}\right)\right]^{1 / 2},} & \text { for } i<j, \\ {\left[S_{w}\left(\omega_{0}\right)\left(\left[\mathbf{S}^{-1}\left(\omega_{0}\right)\right]_{i i}-\left[\widetilde{\mathbf{S}}_{j}^{-1}\left(\omega_{0}\right)\right]_{i-1, i-1}\right)\right]^{1 / 2},} & \text { for } i>j .\end{cases}
$$

Proof: Without loss of generality, we consider the case $j=N$ (for any other $j \neq N$, we can transform the problem to the case $j=N$ via a simple reordering of rows and columns). Subtracting the diagonal elements of $\mathbf{S}^{-1}(\omega)$ in (9) from those of $\widetilde{\mathbf{S}}_{j}^{-1}(\omega)$ in (3), we obtain

$$
\left[\mathbf{S}^{-1}(\omega)\right]_{i i}-\left[\widetilde{\mathbf{S}}_{j}^{-1}(\omega)\right]_{i i}=\frac{\left[\mathbf{G}^{T} \mathbf{G}\right]_{i i}-\left[\widetilde{\mathbf{G}}_{N}^{T} \widetilde{\mathbf{G}}_{N}\right]_{i i}}{S_{w}(\omega)}
$$

Also, since $\left[\mathbf{G}^{T} \mathbf{G}\right]_{i i}=\sum_{k} g_{k i}^{2}$ and $\left[\widetilde{\mathbf{G}}_{N}^{T} \widetilde{\mathbf{G}}_{N}\right]_{i i}=\sum_{k \neq N} g_{k i}^{2}$, we have that

$$
\left[\mathbf{G}^{T} \mathbf{G}\right]_{i i}-\left[\widetilde{\mathbf{G}}_{N}^{T} \widetilde{\mathbf{G}}_{N}\right]_{i i}=g_{N i}^{2}
$$




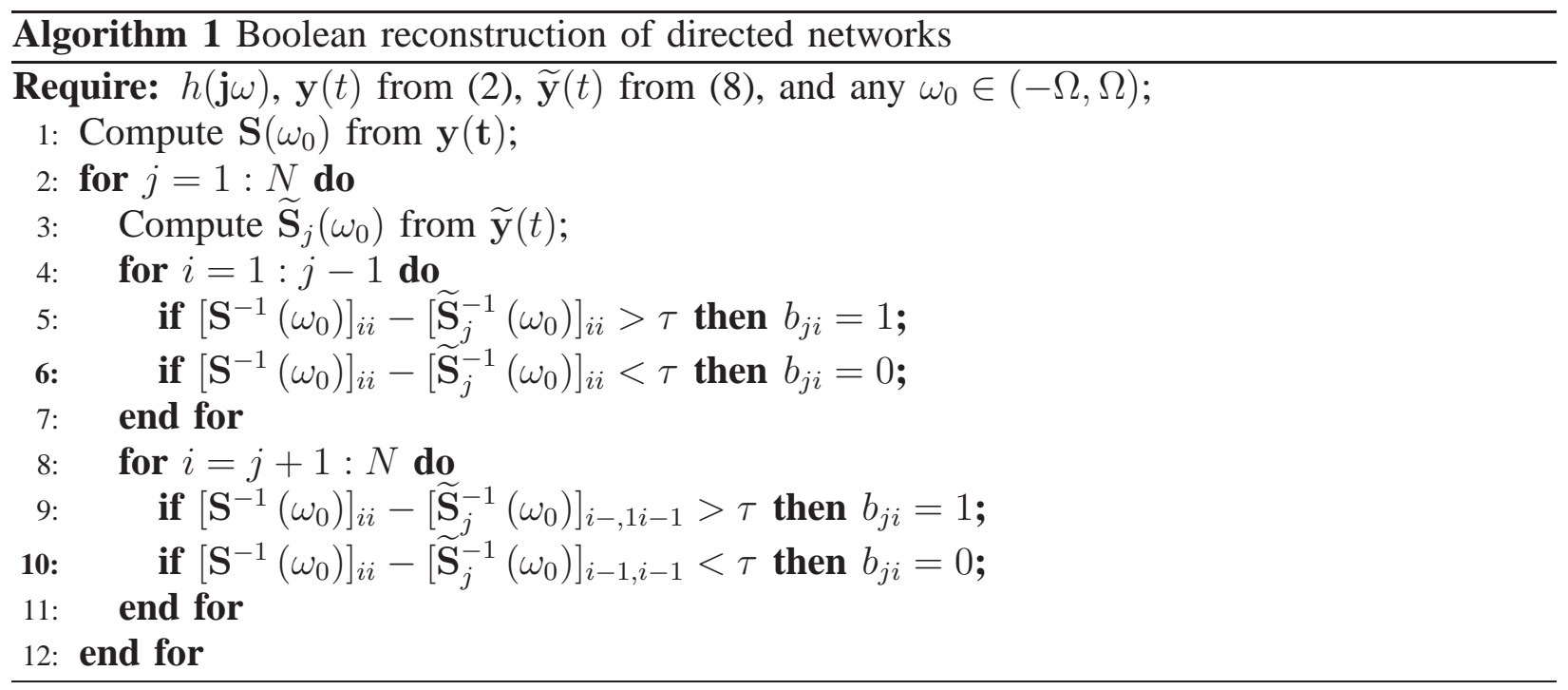

for any $i<N$. The same analysis holds for $j \neq N$. Hence, we can recover the entries $g_{j i}$, for $i<j$, as stated in our Theorem. Notice also that, for $j \neq N$ and $i>j$, we must use the entry $\left[\widetilde{\mathbf{S}}_{j}^{-1}(\omega)\right]_{i-1, i-1}$ in $(10)$, to take into account that $\widetilde{\mathbf{S}}_{j}(\omega)$ is an $(N-1) \times(N-1)$ matrix associated to the dynamics grounded at node $v_{j}$.

\section{A. Boolean Reconstruction of Directed Networks}

Theorem 7 allows us to reconstruct the Boolean structure of an unknown directed network if we have access to the matrices of CPSD's, $\mathbf{S}\left(\omega_{0}\right)$ and $\widetilde{\mathbf{S}}_{j}\left(\omega_{0}\right)$, for any $\omega_{0}$ in the excitation frequency interval $(-\Omega, \Omega)$. In particular, one can verify the existence of a directed edge $(i, j)$ by checking the condition $g_{j i}>0$, where $g_{j i}$ is computed from (10). In practice, the CPSD's $\mathbf{S}\left(\omega_{0}\right)$ and $\widetilde{\mathbf{S}}_{j}\left(\omega_{0}\right)$ are empirically computed from the stochastic outputs of the network, $\mathbf{y}(t)$ and $\widetilde{\mathbf{y}}(t)$; therefore, they are subject to numerical errors. Hence, in the implementation, one should relax the condition $g_{j i}>0$ to $g_{j i}>\tau$, where $\tau$ is a small threshold used to account for numerical precision.

Based on Theorem 7 we propose Algorithm 1 to find the Boolean representation of G, denoted by $\mathbf{B}(\mathbf{G})$, when a directed dynamical network is excited by an input noise of unknown PSD.

Algorithm 1 incurs the following computational cost:

i) It computes the cross-correlation functions for all the $N^{2}$ pairs of outputs in (2). For each one of the $N$ grounded dynamics in (8), the algorithm also computes $N^{2}$ pairs of cross- 
correlation functions, resulting in a total of $N^{3}$. To compute these cross-correlations we use time series of length $L$. Since each each cross-correlation takes $\mathcal{O}\left(L^{2}\right)$ operations, we have a total of $\mathcal{O}\left(N^{3} L^{2}\right)$ operations to compute all the required cross-correlations.

ii) Algorithm 1 computes the FFT of all the $(N+1) N^{2}$ cross-correlation function of length $L$ in $(i)$ at a particular frequency $\omega_{0} \in(-\Omega, \Omega)$. Since computing the FFT at a single frequency takes $\mathcal{O}(L)$ operations, we have a total of $\mathcal{O}\left(N^{3} L\right)$ operations to compute the CPSD's matrices $\mathbf{S}\left(\omega_{0}\right)$ and $\widetilde{\mathbf{S}}_{j}\left(\omega_{0}\right)$, for all $j=1, \ldots, N$.

iii) Our algorithm also needs to compute the inverse of $\mathbf{S}(\omega)$ and $\widetilde{\mathbf{S}}_{j}(\omega)$. Since each inversion takes $\mathcal{O}\left(N^{3}\right)$, we have a total of $\mathcal{O}\left(N^{4}\right)$ operations to compute the inverses of all the $N+1$ matrices involved in our computations.

Therefore, the total computational cost of our algorithm is $\mathcal{O}\left(N^{4}+N^{3} L^{2}\right)$. In the next subsection, we extend Algorithm 1 to reconstruct the exact connectivity matrix G.

\section{B. Exact Reconstruction of Directed Networks}

Apart from a Boolean reconstruction of $\mathbf{G}$, we can also compute the weights of the edges in the network if we know one eigenvalue-eigenvector pair $(\lambda, \mathbf{u})$ of $\mathbf{G}$, as follows. This is the case of $\mathrm{G}$ being, for example, a Laplacian matrix (since $\mathrm{G} \mathbf{1}_{N}=0$, in this case), or the adjacency matrix of a $d$-regular graph (since $\mathbf{G} \mathbf{1}_{N}=d \mathbf{1}_{N}$ ). In these cases, we use Lemma 7 to find the value of $S_{w}\left(\omega_{0}\right)$ at a particular frequency $\omega_{0} \in(-\Omega, \Omega)$. For example, in the case of G being a Laplacian, we have the following result:

Corollary 8: Consider the networked dynamical system in (2), when $\mathbf{G}=-L_{\mathcal{D}}$, where $L_{\mathcal{G}}$ is the Laplacian matrix of a directed graph $\mathcal{D}$. Then, under assumptions (A1)-(A2), the PSD of the input noise, $S_{w}(\omega)$, can be computed as

$$
S_{w}(\omega)=\frac{N}{\left(\mathbf{1}^{T} \mathbf{S}^{-1}(\omega) \mathbf{1}\right)|h(\mathbf{j} \omega)|^{2}} .
$$

Proof: This result can be directly obtained from Lemma 5 taking into account that the eigenpair $(\lambda, \boldsymbol{u})$ for the Laplacian matrix is $(0, \mathbf{1})$.

In general, we can reconstruct the weights of directed edges in a dynamical network using Algorithm 2

Remark 9: It is worth remarking that the reconstruction methods proposed in the paper do not require the entire power spectra for $\mathbf{S}(\omega)$ or $S_{w}(\omega)$, but only the values of these spectral 


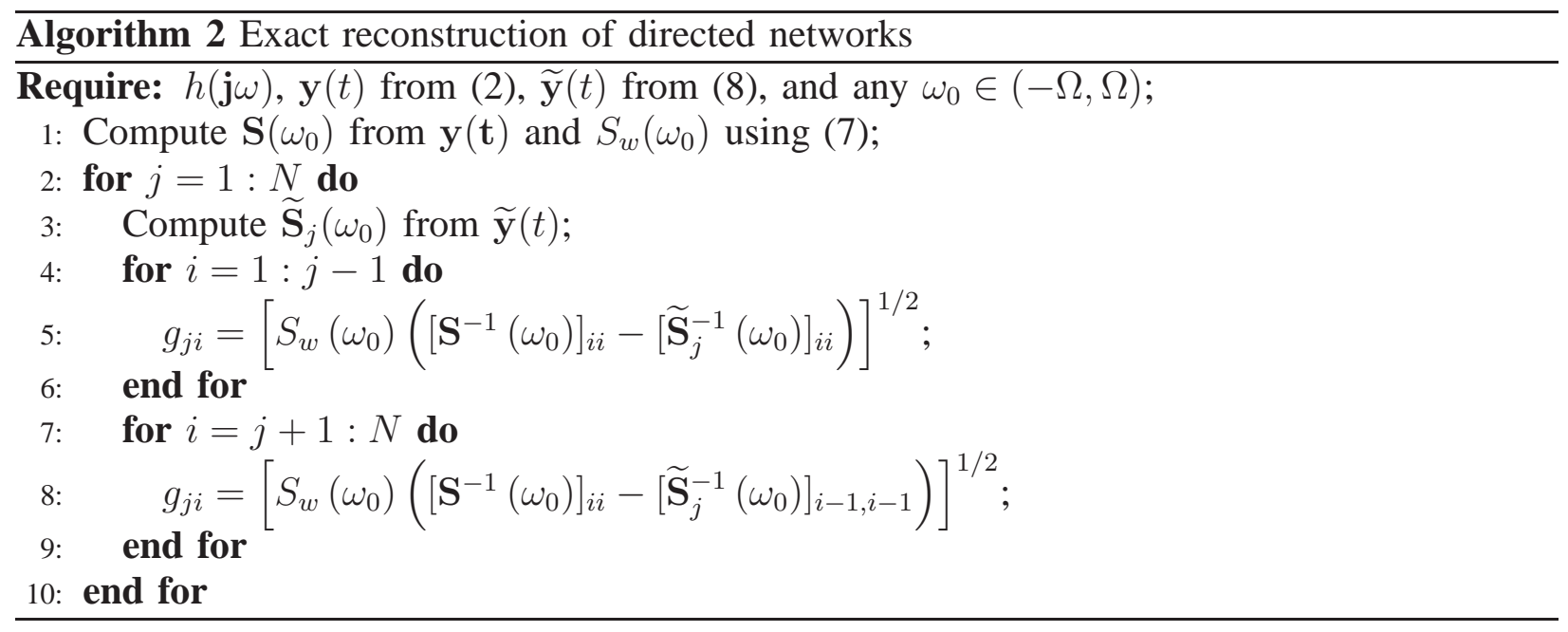

densities at any frequency $\omega_{0} \in(-\Omega, \Omega)$. This dramatically reduce the computational complexity of the reconstruction.

There are two particular types of networks, namely, undirected and nonreciprocal networks, in which the computational cost of reconstruction can be drastically reduced.

\section{Exact Reconstruction of Undirected Networks}

Consider Problem 1, when the connectivity matrix G is an unknown (possibly weighted) symmetric matrix. Then, when an eigenpair $(\lambda, \boldsymbol{u})$ is known, we can find the exact structure of the network from the matrix of CPSD's, $\mathbf{S}(\omega)=\left[S_{y_{i} y_{j}}(\omega)\right]_{1 \leq i, j \leq N}$, and the nodal transfer function, $h(\mathbf{j} \omega)=c^{T}\left(\mathbf{j} \omega I_{n}-A\right)^{-1} b$, using the following result:

Theorem 10: Consider the networked dynamical system (2), when $\mathbf{G}=\mathbf{G}^{T}$. Then, under assumptions (A1)-(A2), we have that

$$
\mathbf{G}=\operatorname{Re}\left\{h^{-1}\left(\mathbf{j} \omega_{0}\right)\right\} I_{N}+\left(\mathbf{S}^{-1}\left(\omega_{0}\right) S_{w}\left(\omega_{0}\right)-\operatorname{Im}^{2}\left\{h^{-1}\left(\mathbf{j} \omega_{0}\right)\right\} I_{N}\right)^{1 / 2},
$$

for any $\omega_{0} \in(-\Omega, \Omega)$. 
Proof: From Lemma 4, we obtain the following for $\mathbf{G}^{T}=\mathrm{G}$ :

$$
\begin{aligned}
\mathbf{S}^{-1}(\omega) S_{w}(\omega) & =\frac{I_{N}}{|h(\mathbf{j} \omega)|^{2}}+\mathbf{G}^{2}-\frac{\mathbf{G}}{h^{*}(\mathbf{j} \omega)}-\frac{\mathbf{G}}{h(\mathbf{j} \omega)} \\
& =\mathbf{G}^{2}-2 \operatorname{Re}\left\{h^{-1}(\mathbf{j} \omega)\right\} \mathbf{G}+I_{N}\left(\operatorname{Im}^{2}\left\{h^{-1}(\mathbf{j} \omega)\right\}+\operatorname{Re}^{2}\left\{h^{-1}(\mathbf{j} \omega)\right\}\right) \\
& =\left(\mathbf{G}-\operatorname{Re}\left\{h^{-1}(\mathbf{j} \omega)\right\} I_{N}\right)^{2}+\operatorname{Im}^{2}\left\{h^{-1}(\mathbf{j} \omega)\right\} I_{N},
\end{aligned}
$$

from which we easily derive the statement of our Theorem.

Based on Theorem 10, we can reconstruct the connectivity matrix $\mathrm{G}=\mathrm{G}^{T}$ when we know an eigenpair of G. The input PSD in (11) can be computed using Lemma 5. Notice that this algorithm does not require grounding the dynamics of the network, resulting in a reduced computational cost. In particular, the computational cost is dominated by the computation of $\mathbf{S}\left(\omega_{0}\right)$, which requires $\mathcal{O}\left(N^{2} L^{2}\right)$ operations, and its inversion, which requires $\mathcal{O}\left(N^{3}\right)$, resulting in a total cost of $\mathcal{O}\left(N^{2} L^{2}+N^{3}\right)$.

\section{Reconstruction of Non-Reciprocal Networks}

Another particular network structure that does not require grounding in the reconstruction method is the so-called nonreciprocal directed networks. In a nonreciprocal network, having an edge $\left(v_{j}, v_{i}\right) \in \mathcal{E}_{d}$ implies that $\left(v_{i}, v_{j}\right) \notin \mathcal{E}_{d}$. In other words, the connectivity matrix of a purely unidirectional network satisfies $\operatorname{Tr}\left(\mathrm{G}^{2}\right)=\sum_{i} \sum_{j} g_{i j} g_{j i}=0$, since, if $g_{i j} \neq 0$, then $g_{i j}=0$ (and assuming there are no self-loops in the network).

The following Theorem allows the Boolean reconstructing of a nonreciprocal network. Moreover, if we have access to an eigenpair of $\mathrm{G}$, this Theorem could be used to perform an exact reconstruction without grounding the dynamics of the network.

Theorem 11: Consider the networked dynamical system (2), with a connectivity matrix satisfying $\mathbf{G} \geq 0$ (nonnegativity) and $\operatorname{Tr}\left(\mathbf{G}^{2}\right)=0$ (nonreciprocity). Then, under assumptions (A1)-(A2), we have that

$$
g_{i j}=\max \left\{S_{w}(\omega)\left(\frac{\left[\operatorname{Im}\left\{\mathbf{S}^{-1}(\omega)\right\}\right]_{i j}}{\operatorname{Im}\left\{h^{-1}(\mathbf{j} \omega)\right\}}\right), 0\right\},
$$

for $1 \leq i \neq j \leq N$. 
Proof: Under purview of Lemma 4, we obtain

$$
\mathbf{S}^{-1}(\omega) S_{w}(\omega)=\frac{I_{N}}{|h(\mathbf{j} \omega)|^{2}}+\mathbf{G}^{T} \mathbf{G}-\frac{\mathbf{G}}{h^{*}(\mathbf{j} \omega)}-\frac{\mathbf{G}^{T}}{h(\mathbf{j} \omega)} .
$$

Taking the imaginary parts, we obtain

$$
\operatorname{Im}\left\{\mathbf{S}^{-1}(\omega) S_{w}(\omega)\right\}=\operatorname{Im}\left\{-\frac{\mathbf{G}}{h^{*}(\mathbf{j} \omega)}-\frac{\mathbf{G}^{T}}{h(\mathbf{j} \omega)}\right\}=\operatorname{Im}\left\{h^{-1}(\mathbf{j} \omega)\right\}\left(\mathbf{G}-\mathbf{G}^{T}\right),
$$

which entails

$$
\mathbf{G}-\mathbf{G}^{T}=\frac{S_{w}(\omega)}{\operatorname{Im}\left\{h^{-1}(\mathbf{j} \omega)\right\}} \operatorname{Im}\left\{\mathbf{S}^{-1}(\omega)\right\}
$$

Given that $\mathbf{G} \geq 0$ and the network is nonreciprocal, if $\left[\mathbf{G}-\mathbf{G}^{T}\right]_{i j}>0$, then $g_{i j}>0$ and $g_{j i}=0$. If $\left[\mathbf{G}-\mathbf{G}^{T}\right]_{i j}<0$, then $g_{i j}=0$ and $g_{j i}>0$. Finally, if $\left[\mathbf{G}-\mathbf{G}^{T}\right]_{i j}=0$, then no directed edge between $v_{i}$ and $v_{j}$ exists. These three conditional statements can be condensed into (12).

Using this Theorem, we can find the the Boolean representation of $\mathbf{G}, \mathbf{B}(\mathbf{G})=\left[b_{i j}\right]$, as follows,

$$
b_{i j}= \begin{cases}1, & \text { if } \frac{\left[\operatorname{Im}\left\{\mathbf{S}^{-1}\left(\omega_{0}\right)\right\}\right]_{i j}}{\operatorname{Im}\left\{h^{-1}\left(\mathbf{j} \omega_{0}\right)\right\}}>0 \\ 0, & \text { otherwise }\end{cases}
$$

where $\omega_{0} \in(-\Omega, \Omega)$. Moreover, if an eigenvalue eigenvector pair of $\mathbf{G}$ is known, we can recover $S_{w}\left(\omega_{0}\right)$ using Lemma 5, which allows us to recover the value of $g_{i j}$ directly from 12 , Following the analysis of previous algorithms, the computational cost of the reconstruction of a nonreciprocal directed network is $\mathcal{O}\left(N^{2} L^{2}+N^{3}\right)$.

\section{CONCLUSIONS}

In this paper, we have addressed the problem of identifying the topology of an unknown directed network of LTI systems stimulated by a wide-sense stationary noise of unknown power spectral density. We have proposed several reconstruction algorithms based on the power spectral properties of the network response to the noise. Our first algorithm reconstructs the Boolean structure of a directed network based on a series of grounded dynamical responses. Our second algorithm recovers the exact structure of the network (including edge weights) when an eigenvalue-eigenvector pair of the connectivity matrix is known. This algorithm is useful, for 
example, when the connectivity matrix is a Laplacian matrix or the adjacency matrix of a regular graph. Apart from general directed networks, we have also proposed more computationally efficient algorithms for both directed nonreciprocal networks and undirected networks.

\section{REFERENCES}

[1] R. Bonneau, D. Reiss, P. Shannon, M. Facciotti, L. Hood, N. Baliga, and V. Thorsson, "The inferelator: an algorithm for learning parsimonious regulatory networks from systems-biology data sets de novo," Genome Biology, vol. 7, no. 5, p. R36, 2006.

[2] F. Geier, J. Timmer, and C. Fleck, "Reconstructing gene-regulatory networks from time series, knock-out data, and prior knowledge,” BMC Systems Biology, vol. 1, no. 1, p. 11, 2007.

[3] M. Bansal, V. Belcastro, A. Ambesi-Impiombato, and D. Di Bernardo, "How to infer gene networks from expression profiles," Molecular Systems Biology, vol. 3, no. 1, 2007.

[4] A. Julius, M. Zavlanos, S. Boyd, and G. Pappas, "Genetic network identification using convex programming," Systems Biology, IET, vol. 3, no. 3, pp. 155-166, 2009.

[5] S. Boccaletti, M. Ivanchenko, V. Latora, A. Pluchino, and A. Rapisarda, "Detecting complex network modularity by dynamical clustering," Physical Review E, vol. 75, no. 4, p. 045102, 2007.

[6] M. Timme, "Revealing network connectivity from response dynamics," Physical Review Letters, vol. 98, no. 22, p. 224101, 2007.

[7] D. Napoletani, T. Sauer et al., "Reconstructing the topology of sparsely connected dynamical networks," Physical Review E, vol. 77 , no. 2 , p. $26103,2008$.

[8] R. Mantegna and H. Stanley, An Introduction to Econophysics: Correlations and Complexity in Finance. Cambridge University Press, 2000.

[9] C. D. Michener and R. R. Sokal, “A quantitative approach to a problem in classification,” Evolution, pp. 130-162, 1957.

[10] D. Marinazzo, M. Pellicoro, and S. Stramaglia, "Kernel method for nonlinear granger causality," Physical Review Letters, vol. 100 , no. 14 , p. $144103,2008$.

[11] C. W. Granger, "Investigating causal relations by econometric models and cross-spectral methods," Econometrica, pp. 424-438, 1969.

[12] F. R. Bach and M. I. Jordan, "Learning graphical models for stationary time series," IEEE Transactions on Signal Processing, vol. 52, no. 8, pp. 2189-2199, 2004.

[13] E. Candes, M. Wakin, and S. Boyd, "Enhancing sparsity by reweighted $\ell_{1}$ minimization," Journal of Fourier Analysis and Applications, vol. 14, no. 5, pp. 877-905, 2008.

[14] J. Gonçalves and S. Warnick, "Necessary and sufficient conditions for dynamical structure reconstruction of LTI networks," IEEE Transactions on Automatic Control, vol. 53, no. 7, pp. 1670-1674, 2008.

[15] Y. Yuan, G. Stan, S. Warnick, and J. Goncalves, "Robust dynamical network structure reconstruction,” Automatica, 2011.

[16] D. Materassi and G. Innocenti, "Unveiling the connectivity structure of financial networks via high-frequency analysis," Physica A, vol. 388, no. 18, pp. 3866-3878, 2009.

[17] — " "Topological identification in networks of dynamical systems," IEEE Transactions on Automatic Control, vol. 55, no. 8 , pp. $1860-1871,2010$. 
[18] D. Materassi and M. Salapaka, "On the problem of reconstructing an unknown topology via locality properties of the wiener filter," IEEE Transactions on Automatic Control, vol. 57, no. 7, pp. 1765-1777, 2012.

[19] M. Nabi-Abdolyousefi and M. Mesbahi, "Sieve method for consensus-type network tomography," IET Control Theory \& Applications, vol. 6, no. 12, pp. 1926-1932, 2012.

[20] —_ "Network identification via node knockout," IEEE Transactions on Automatic Control, vol. 57, no. 12, pp. 3214-3219, 2012.

[21] D. West, Introduction to Graph Theory. Prentice-Hall, 2001, vol. 2.

[22] N. Biggs, Algebraic Graph Theory. Cambridge Univ Pr, 1993.

[23] D. R. Brillinger, Time series: data analysis and theory. Siam, 1981, vol. 36.

[24] D. Tylavsky and G. Sohie, "Generalization of the matrix inversion lemma," Proceedings of the IEEE, vol. 74, no. 7, pp. 1050-1052, 1986.

[25] S. Shahrampour and V. M. Preciado, "Reconstruction of directed networks from consensus dynamics," in IEEE American Control Conference, 2013. 